Article

\title{
Modelling of Reverse Flows in a Mechanical Seal
}

\author{
Noël Brunetière* \\ Institut Pprime - UPR 3346 - CNRS - Université de Poitiers - ENSMA \\ 86962 FUTUROSCOPE - France \\ "Corresponding author: noel.brunetiere@univ-poitiers.fr \\ ( Manuscript received 26 October 2015; accepted 20 December 2015; published 30 April 2016 ) \\ (Presented at the International Tribology Conference Tokyo 2015, 16-20 September, 2015 )

\begin{abstract}
The present paper describes a two-phase flow model used to study backward pumping of the low pressure fluid in the sealing interface of a mechanical seal. The Reynolds equation for a homogeneous mixture is solved as well as a transport equation used to determine the ratio of both fluids at each point. Several examples are presented to show that the second function of a seal (prevent the contamination of the process fluid by the operating environment) is not always insured because of possible reverse flows. The behavior of the seal can be greatly affected when it is partially lubricated by the low pressure fluid.
\end{abstract}

Keywords: mechanical seal, two phase flow, backward pumping, reverse flow

\section{Introduction}

The sealing of rotating machines such as pumps or compressor is generally insured by mechanical face seals. They are basically composed of two flat annular rings, one being linked to the housing and the other to the shaft of the machine, maintained in contact to prevent leakage of the process fluid to the atmosphere. The secondary function of a mechanical seal is to prevent the contamination of the process fluid by the operating environment [1]. The fluid transfer or fluid leakage takes place because the surfaces are not perfectly flat and smooth leading to the generation of a fluid film between the sliding surfaces. This film helps in reducing friction and allows a safe operation without intensive wear.

In 1961, Denny [2] made a pionniring experimental work on mechanical seal. He presented lekage measurements but found that a reverse flow or pumping flow can be observed in some situations. Reverse flow means the fluid located on the low pressure side of the seal is pumped into the contact. A few years later, Nau [3] performed a review and underlined that reverse pumping or inwards pumping has been observed by several authors. More recently, Lebeck [4] stated that reverse pumping can affect the leakage of a seal.

This mechanism is known to allow hermetic sealing in lip seals [5] but has been rarely studied in mechanical seals. Even if reverse flow is evaluated, the objective here is to identify the amount of fluid in the contact and its effect on the performance. This topic is of interest when the environnemental fluid can polute the process or reduce the life expectancy of the seal.

To study reverse flow it is necessary to consider a two phase or two components flow with one components coming from the outer radius and the other from the inner radius of the seal. In the book of Wallis [6], two solutions are proposed to model two phase flow. One of them is the separated flow approach where each phase has its own velocity field. This technic has been used by Szeri [7] to analyse the fluid flow of emulsions in tribological contacts. The other solution consists in assuming a homogeneous mixture where each components has the same velocity. This approach has been widely used to study vaporization in mechanical face seals [8-11]. The problem is then to determine the relative mass fraction of each phase. In the case of boiling this is done using the energy equation which control the amount of fluid vaporized [8-10]. The case of two-phase flow due to different fluids at the seal boundaries has been rarely studied. Lebeck [12] studied this problem in the case of a grooved seal which was designed to ensure a backward pumping. His model is based on the homogeneous fluid assumption. To solve the problem, he derived two mass flow equations, one for each phase. A very similar method was employed later by Wang et al [11] to sudy vaporization.

In the present paper a different approach is proposed. The Reynolds equation is used to determine the fluid flow of the homogenous mixture. An additional transport equation is used to calculate the local mass fraction of each phase. This model is then applied to a mechanical seal to analyze the magnitude and the effect of the 
reverse flow on the sealing performance.

\section{Theoretical model}

The general configuration of the problem is presented on Figure 1. The problem is supposed to be stationary. The seal is composed of a flat rotating ring. Its angular speed is $\omega$. The upper ring is stationary but can moves axially in order to reach an equilibrium position. The film thickness is given by:

$$
h=h_{0}+f(x, y)
$$

where $h_{0}$ is the centerline clearance and $f$ a function describing the height variation of the stator. The fluid between the seal faces is a homogenous mixture of liquid and gas. Its behavior is described in the way proposed by Brunetière and Wang [13]. The density of the mixture is expressed:

$$
\rho=\frac{1}{\frac{1-\lambda}{\rho_{l}}+\frac{\lambda}{\rho_{g}}}
$$

where $\lambda$ is the gas mass fraction. $\rho_{1}$ is the liquid density and $\rho_{g}$ the gas density given by the ideal gas law:

$$
\rho_{g}=\frac{p}{r_{g} T}
$$

$p$ is the fluid pressure, $T$ the temperature and $r_{g}$ the ideal gas constant of the considered fluid. The viscosity of the mixture is expressed in a similar way as the density:

$$
\mu=\frac{1}{\frac{1-\lambda}{\mu_{l}}+\frac{\lambda}{\mu_{g}}}
$$

The viscosity law choice can be discussed but this is not the topic of the present work. Another law can be easily used. This approach allows describing film rupture in a continuous fashion without any switch function as in usual cavitation model [13].

The fluid flow between the seal surfaces is governed by the Reynolds equation:

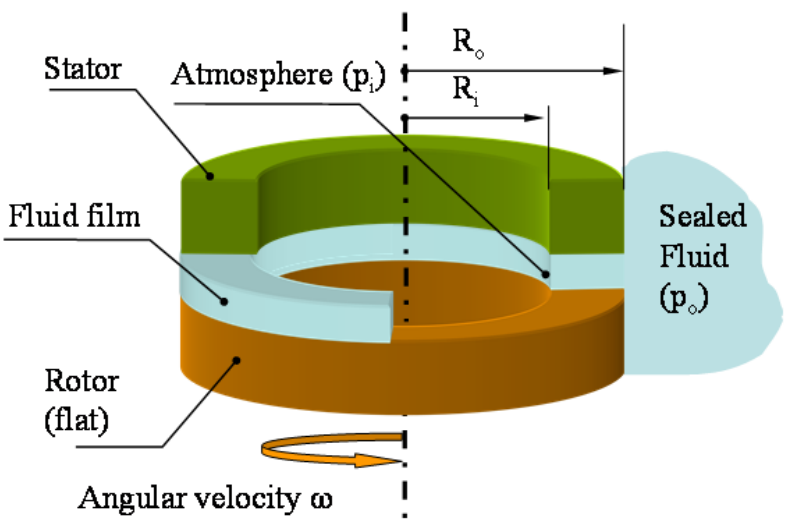

Fig. 1 Geometrical configuration of the studied problem

$$
\begin{aligned}
& \frac{\partial}{\partial x}\left(\frac{\rho h^{3}}{12 \mu} \frac{\partial p}{\partial x}\right)+\frac{\partial}{\partial y}\left(\frac{\rho h^{3}}{12 \mu} \frac{\partial p}{\partial y}\right)= \\
& -\frac{y \omega}{2} \frac{\partial \rho h}{\partial x}+\frac{x \omega}{2} \frac{\partial \rho h}{\partial y}+\frac{\partial}{\partial x}\left(\frac{\rho^{2} h^{3} \omega^{2} x}{36 \mu}\right) \\
& +\frac{\partial}{\partial y}\left(\frac{\rho^{2} h^{3} \omega^{2} y}{36 \mu}\right)
\end{aligned}
$$

It is assumed that the density and the viscosity of the fluid do not vary in the through film direction and that the surface roughness has no effect on the flow. The two last terms correspond to the centrifugal inertia contribution on the fluid flow. When high speeds are considered (as in the present paper), it is mandatory to include this effect. It should be noted that the Reynolds equation is formulated in Cartesian coordinate in order to be solved by the finite element method.

It is now necessary to define an equation which will govern a species concentration transport in the film. If the variable is a dimensionless mass fraction $c$, the steady state transport equation is given by [14]:

$$
\frac{\partial \rho c u}{\partial x}+\frac{\partial \rho c v}{\partial y}+\frac{\partial \rho c w}{\partial z}=D\left[\frac{\partial^{2} \rho c}{\partial x^{2}}+\frac{\partial^{2} \rho c}{\partial y^{2}}+\frac{\partial^{2} \rho c}{\partial z^{2}}\right]
$$

where $u, v$ and $w$ are the fluid velocity components and $D$ a diffusion coefficient. It is assumed that there is no diffusion. It means there will be no mixing if there is no fluid motion. It is finally assumed that the mass fraction $c$ is constant through the film thickness. Equation (6) can be integrated in the $z$-direction leading to the following equation:

$$
q_{x} \frac{\partial c}{\partial x}+q_{y} \frac{\partial c}{\partial y}=0
$$

In this simple equation, $q_{x}$ and $q_{y}$ are respectively the mass flow rate in the $x$ and $y$ directions. They can be expressed:

$$
\begin{aligned}
& q_{x}=\int_{0}^{h} u \mathrm{~d} z=-\frac{y \omega}{2} \rho h+\frac{\rho h^{3}}{12 \mu}\left(\frac{\rho \omega^{2} x}{3}-\frac{\partial p}{\partial x}\right) \\
& q_{y}=\int_{0}^{h} v \mathrm{~d} z=\frac{x \omega}{2} \rho h+\frac{\rho h^{3}}{12 \mu}\left(\frac{\rho \omega^{2} y}{3}-\frac{\partial p}{\partial y}\right)
\end{aligned}
$$

Finally a simple elastic contact model is used to avoid unrealistic film thickness values. The contact pressure $p_{c}$ is calculated with a simplified Greenwood and Williamson approach [15]:

$$
p_{c}(h)=K e^{-\frac{h}{2 \sigma}}
$$

where $K$ is a stiffness parameter depending on the material properties and on the roughness statistical properties and $\sigma$ is the standard deviation of the asperities summit height.

\section{Numerical solution}

The problem is discretized using the finite element method. This procedure was chosen because it can be 
easily applied to complex geometrical problems such as spiral groove seals. The finite element solution of the compressible Reynolds equation will not be addressed here. It has been done in many papers [16-18]. The boundary conditions for the Reynolds equation are imposed pressure at the inner and outer radii:

$$
\begin{aligned}
& p\left(R_{0}\right)=p_{0} \\
& p\left(R_{i}\right)=p_{i}
\end{aligned}
$$

If the domain is periodical in the circumferential direction, a periodicity condition is used:

$$
p\left(\theta+\theta_{p}\right)=p(\theta)
$$

where $\theta_{p}$ is the angle of the angular period.

The transport equation (7) is written in an integral form using the weighted residuals approach [19]:

$$
\int_{\Omega} w_{i}\left(q_{x} \frac{\partial N_{j}}{\partial x}++q_{y} \frac{\partial N_{j}}{\partial y}\right) c_{j}=0
$$

In this equation, $w_{i}$ is the weighing function of the node $\# i$ and $N_{j}$ is the interpolation and shape function of the node $\# j$. Since this equation is a pure transport equation, the standard Galerkin method consisting in using $w_{i}=N_{i}$ cannot be used otherwise severe numerical oscillations will be obtained [19]. A solution is to use an upwind scheme which considers the direction of the flow in the weighting function. The streamline upwind Petrov-Galerking (SUPG) method is used in the present work. This method is based on the following weighting function $[20,21]$ :

$$
w_{i}=N_{i}+\alpha \frac{L_{e}}{2 q}\left(q_{x} \frac{\partial N_{i}}{\partial x}+q_{y} \frac{\partial N_{i}}{\partial y}\right)
$$

where $q$ is the norm of the mass flow rate, $L_{e}$ is the characteristic length of the considered element and $\alpha$ is an upwinding coefficient. If this coefficient is set to zero, the standard Bubnov-Galerking method is obtained. The transport equation being a pure advection equation, $\alpha$ must be set to 1, corresponding to full uwpwinding.

The value of the concentration $c$ is imposed if the flow is inlet otherwise, its value is unknown and must be calculated as for other points located inside of the domain:

$$
\begin{cases}q_{x} \cdot n_{x}+q_{y} \cdot n_{y}<0 & c=\text { imposed } \\ q_{x} \cdot n_{x}+q_{y} \cdot n_{y} \geq 0 & c=\text { unknown }\end{cases}
$$

Here $n_{x}$ and $n_{y}$ are the components of the outlet normal of the film domain. At each point of the boundary, it is necessary to check the direction of the flow. Additionally, a periodicity condition is used:

$$
c\left(\theta+\theta_{p}\right)=c(\theta)
$$

To validate the numerical scheme a simple problem has been solved. The two surfaces of the seal are flat and parallel. One quarter of the domain is solved. The operating conditions are given in Table 1 . In this case, the entire outer radius boundary is an inlet frontier because of the pressure differential and the parallel surfaces. The value of the concentration at the inlet is:
Table 1 Data used for the numerical model test

\begin{tabular}{|l|l|}
\hline Parameter & Value \\
\hline$R_{0}(\mathrm{~mm})$ & 33 \\
\hline$R_{\mathrm{i}}(\mathrm{mm})$ & 29 \\
\hline$P_{0}(\mathrm{MPa})$ & 0.2 \\
\hline$P_{\mathrm{i}}(\mathrm{MPa})$ & 0.1 \\
\hline$h_{0}(\mu \mathrm{m})$ & 3 \\
\hline Fluid & Water \\
\hline$\mu(\mathrm{Pa} \cdot \mathrm{s})$ & 0.001 \\
\hline$\rho\left(\mathrm{kg} / \mathrm{m}^{3}\right)$ & 1000 \\
\hline
\end{tabular}

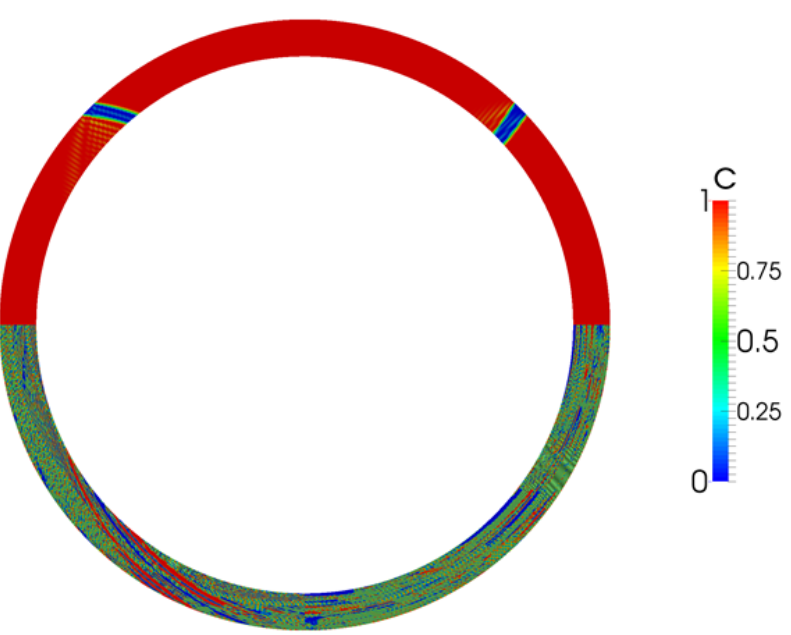

(a)

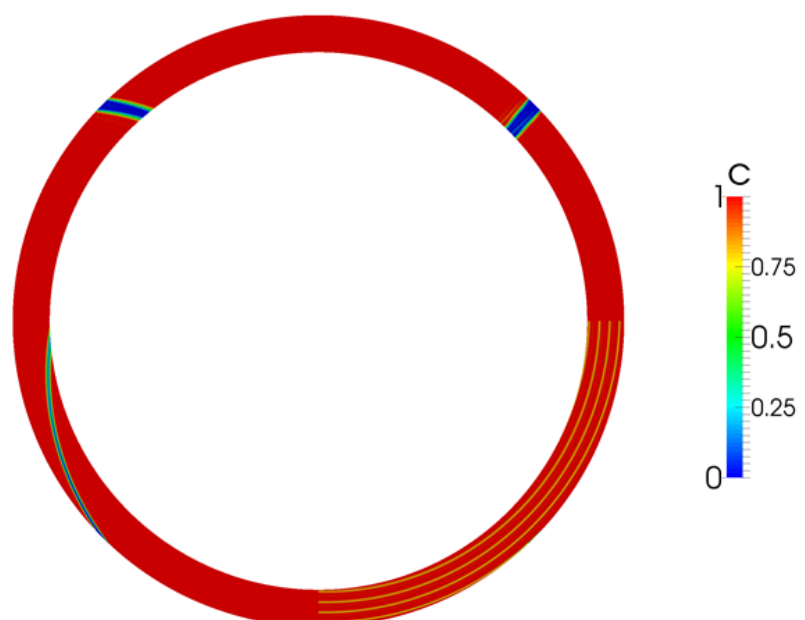

(b)

Fig 2 Distribution of $c$ in the fluid domain when $\omega$ $=$, top right $1 \mathrm{rpm}$, top left $8 \mathrm{rpm}$, bottom left $64 \mathrm{rpm}$, bottom right $512 \mathrm{rpm}$ for a) $\alpha=0$ and b) $\alpha=1$ 


$$
\begin{cases}\left|\theta-\frac{\pi}{4}\right| \leq \frac{\pi}{40} & c=0 \\ \left|\theta-\frac{\pi}{4}\right|>\frac{\pi}{40} & c=1\end{cases}
$$

The results obtained for increasing speed values are presented on Fig. 2 for $\alpha=0$, meaning no upwind scheme and $\alpha=1$. The $c$ distribution can be seen as the fluid particle trajectory. For the smallest value of $\omega$, the fluid flows mainly in the radial direction due to the radial pressure differential. When the rotating speed is increased, the fluid particles tend to describe a spiral with an increasing angle compared to the radial direction. If a standard Bubnov-Galerkin scheme $(\alpha=0)$ is used, severe numerical oscillations are observed as soon as $\omega$ reaches $64 \mathrm{rpm}$. These oscillations disappeared with the SUPG method. An interesting point is that if the rotation speed is increased again, the number of circumference described by the fluid particles before leaving the sealing interface will increase and ultimately exceed the number of radial nodes. In this case, a kind a mixing will take place since the mesh is not fine enough to capture the exact fluid particles trajectory. This effect should be keep in mind since in a sealing system, the circumferential velocity is sometimes several orders of magnitude higher than the radial speed.

\section{Results}

In this section, the model will be applied to a mechanical seal to analyse the magnitude and the effect of reverse flows.

\subsection{Configuration of the problem}

In this section, a seal with a wavy surface is considered. This type of defect is currently observed in mechanical seals [4,22]. The film thickness equation considering 4 waves is thus:

$$
h=h_{0}+h_{w} \cos (4 \theta)
$$

where $h_{w}$ is the waves amplitude.

The design and operating conditions are given in Table 2. The simulation is performed for speed values ranging from 1 to $16000 \mathrm{rpm}$. Between two steps, the velocity is doubled.

At each speed value, the centerline clearance $h_{0}$ is adjusted in order to ensure axial forces equilibrium on the floating ring of the seal. The force equilibrium equation is given by

$$
\int_{\Omega}\left(p+p_{c}\right) d \Omega=\left[p_{o} B+p_{i}(1-B)\right] \Omega
$$

where $\Omega$ is the area of the sealing interface and $B$ the balance ratio of the seal.

\subsection{Uncoupled results}

In this first set of simulations, it is assumed that the fluid at the inner and outer radii (i. e. LP and HP fluids) of the seal are identical. Thus, the local characteristics of the fluid will not vary if the fluid enters the contact at the inner or outer radius. The Reynolds equation and the
Table 2 Design and operating parameters used in the simulations

\begin{tabular}{|l|l|}
\hline Parameter & Value \\
\hline Outer radius $R_{0}(\mathrm{~mm})$ & 33 \\
\hline Inner radius $R_{i}(\mathrm{~mm})$ & 29 \\
\hline Outer pressure $P_{0}(\mathrm{MPa})$ & 0.2 \\
\hline Inner pressure $P_{i}(\mathrm{MPa})$ & 0.1 \\
\hline Waves half amplitude $\mathrm{h}_{\mathrm{w}}(\mu \mathrm{m})$ & 0.25 \\
\hline Liquid viscosity $\mu_{1}(\mathrm{~Pa} \cdot \mathrm{s})$ & 0.001 \\
\hline Liquid density $\rho_{1}\left(\mathrm{~kg} / \mathrm{m}^{3}\right)$ & 1000 \\
\hline Gas viscosity $\mu_{g}(\mathrm{~Pa} \cdot \mathrm{s})$ & $1.8 \times 10^{-5}$ \\
\hline Gas constant $r_{g}(\mathrm{~J} / \mathrm{kg} / \mathrm{K})$ & 287 \\
\hline Fluid temperature $T(\mathrm{~K})$ & 378 \\
\hline Gas mass fraction $\lambda$ & $10^{-5}$ \\
\hline Rotation speed $\omega(\mathrm{rpm})$ & $1-16384$ \\
\hline Balance ratio $B$ & 0.8 \\
\hline Inter asperity dry friction coefficient $f$ & 0.4 \\
\hline $\begin{array}{l}\text { Standard deviation of the asperities } \\
\text { summit height } \sigma(\mu \mathrm{m})\end{array}$ & 0.025 \\
\hline Roughness stiffness $K(\mathrm{GPa})$ & 0.226 \\
\hline
\end{tabular}

transport equation are thus uncoupled and can be solved simultaneously. The solution procedure is the following. For a given seal clearance, the Reynolds Eq. (5) is solved. Because of the compressibility of the fluid (Eqs. (2) and (3)), an iterative process is used. The contact pressure is then calculated. The forces are determined and the equilibrium Eq. (20) is checked. If equilibrium is not reached, the film thickness is modified according to the Newton's method. When this process is converged, the transport equation (7) can be solved to obtain the distribution of the concentration $c$. $c$ is here the mass fraction of fluid coming from the inner radius. Thus it is zero at the outer radius and one at the inner radius. These values are only imposed at nodes where the fluid enters the contact (see Eq. (16)).

The results are presented on Figures 3 to 6. Figure 3 presents the fluid pressure distribution at four different rotating speed values. For the lowest velocity, the pressure decreases from the outer to the inner radii without significant variations. When the speed is increased, a hydrodynamic pressure peak is generated while a sub-ambient pressure zone is observed. This zone corresponds to the cavitation zone. The magnitude of the pressure peak as well as the extent of the cavitation zone obviously increase with the angular velocity. 


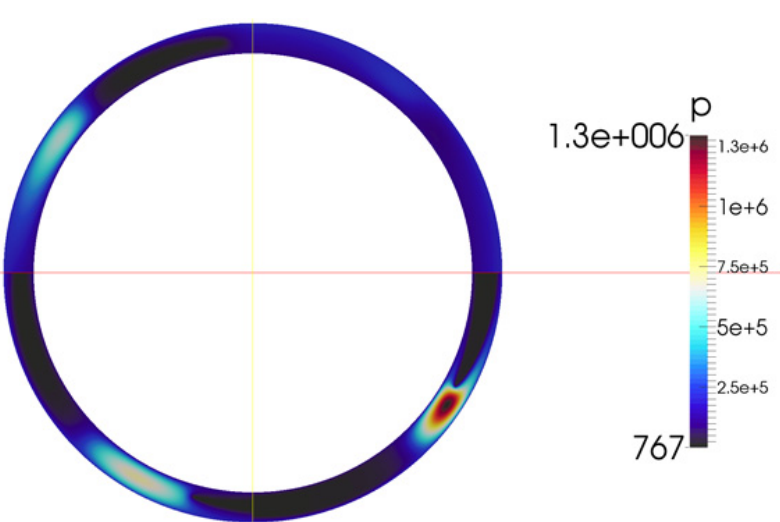

Fig. 3 Pressure distribution $(\mathrm{Pa})$ in the fluid film domain when $\omega=$, top right $1 \mathrm{rpm}$, top left $32 \mathrm{rpm}$, bottom left $1024 \mathrm{rpm}$, bottom right $16384 \mathrm{rpm}$.

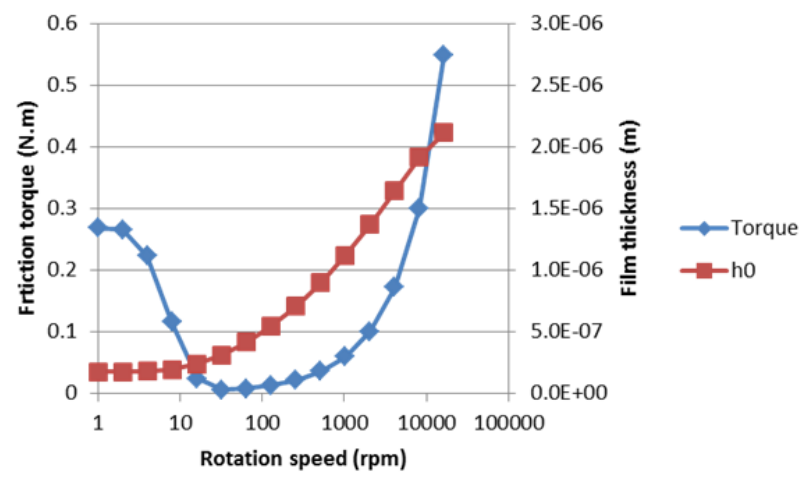

Fig. 4 Friction torque and centerline clearance $h_{0}$ as a function of the rotation speed.

The friction torque and the centerline clearance are presented as a function of the rotating speed on Figure 4. A typical Stribeck curve is obtained. At low speed values, the generated hydrodynamic pressure is not high enough to completely support the closing force applied to the seal. Thus asperity contact occurs leading to a high friction torque value. When the speed is increased, the hydrodynamic pressure generation rises. The film becomes thicker reducing thus the friction due to asperity contact. If the speed is increased again, a minimum friction value is reached, corresponding to the end of asperity contact and of mixed lubrication regime. For higher speed values, the lubrication regime is hydrodynamic and the film thickness and friction torque increase with velocity.

Figure 5 presents the fluid components distribution at four different rotating speed values. The blue color $(c=$ 0 ) indicates that the fluid enters the contact at the outer radius. The red color is for the other radius. At low speed, the sealing interface is mainly blue because it is invaded by the fluid coming from the high pressure side (outer radius, HP fluid). However a small red zone field with fluid from the low pressure side (LP fluid) can be seen close to the inner radius. Some fluid enters the contact from the low pressure side because of sub-ambient pressure values encountered in the sealing interface due

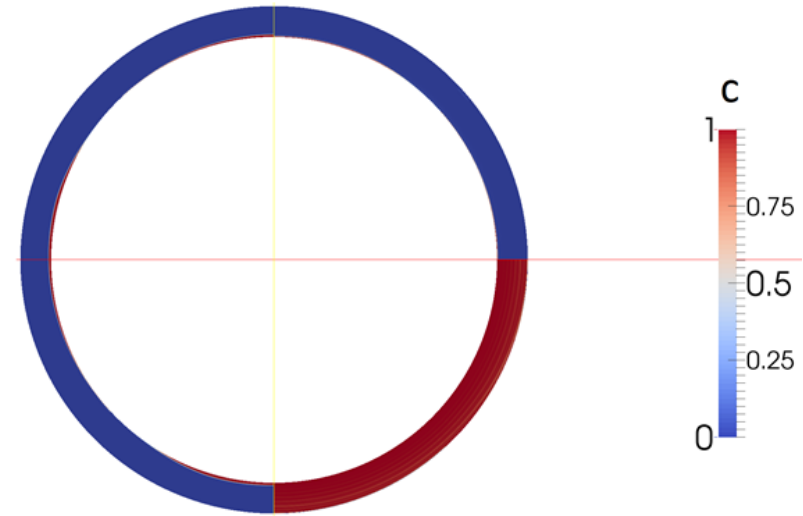

Fig 5 Fluid components distribution in the fluid film domain when $\omega=$, top right $1 \mathrm{rpm}$, top left $32 \mathrm{rpm}$, bottom left $1024 \mathrm{rpm}$, bottom right $16384 \mathrm{rpm} . c=0$ for the fluid coming from the outer radius and $c=1$ for the fluid coming from the inner radius

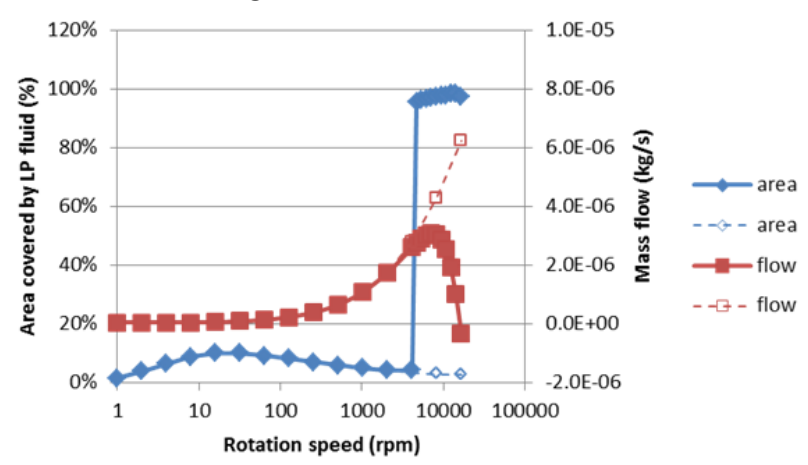

Fig 6 Dimensionless area covered by the LP fluid and the leakage mass flow as a function of the rotation speed. Dashed lines were obtained while neglecting the centrifugal inertia in the Reynolds equation

to cavitation. The fluid is thus suck into the contact. When the speed is increased, the extent of this zone first rises because the size of the cavitation zone is higher. It should be noted that a part of the inner radius boundary is blue. It means that the fluid from the low pressure side enters, makes a small excursion in the sealing interface and then exits the contact before making a full revolution. When the speed is increased again, the size of the red zone tends to decrease. This decrease can be explained in this way. With increasing rotation speed, the fluid particles from the LP side reach more rapidly the exit zone before having time to perform a significant radial excursion. Finally, for the highest speed value, the sealing interface is almost completely filled with the LP fluid. This invasion is due to centrifugal effect which helps the LP fluid to enter the contact.

More quantitative results are presented on Fig. 6 where the dimensionless area covered by the LP fluid as well as the leakage mass flow are plotted as a function of the rotation speed. The dashed lines correspond to simulation results obtained while neglecting the 


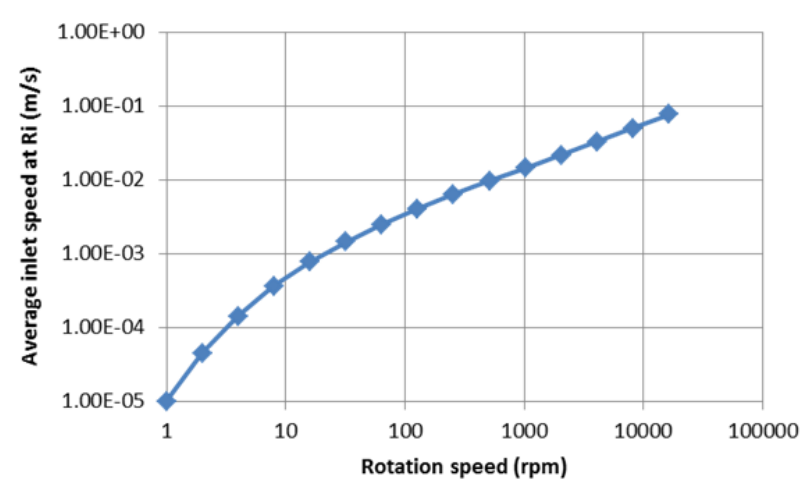

Fig. 7 Averaged inlet speed of the LP fluid as a function of the rotation speed

centrifugal inertia terms. As previously discussed, the area covered by the LP fluid first increases with speed and reaches a maximum value close to $10 \%$ of the sealing area at about $16 \mathrm{rpm}$. Then this area decreases when the speed is raised. At speed value of about $8000 \mathrm{rpm}$, a sharp increase to about $98 \%$ is observed on the dimensionless area. This increase is not observed anymore when centrifugal inertia is neglected. The mass flow exhibits a positive increasing value with speed. A positive value means that the fluid flows from the HP side (outer radius) to the LP side (inner radius). This is why a part of the inner radius is blue indicating that the HP fluid exits the contact in this zone. A sharp reduction and then an inversion of the mass flow rate is observed when the centrifugal inertia becomes significant.

To analyze the decrease in the dimensionless area covered by the LP fluid when the rotating speed reaches $16 \mathrm{rpm}$, the averaged radial inlet speed of the LP fluid at the inner radius is plotted as a function of the rotating speed on Fig. 7. As expected, this inlet speed increases with the rotating speed. But a decrease of the slope is observed close to $16 \mathrm{rpm}$. After this slope change, the radial inlet speed continues to increase but slower than the angular velocity does. Thus the radial excursion of the fluid particles is decreased because the exit area is more rapidly reached due to the high circumferential speed.

This simple set of simulations indicates the magnitude of the sealing area affected by the LP fluid when reverse a flow takes place. The magnitude can be very significant at high speed values when the centrifugal inertia controls the fluid flow. The comparison of the centrifugal inertia term to the pressure terms is given by the following modified Reynolds number [23]:

$$
R e_{c}^{*}=\frac{\rho \omega^{2} R_{0}^{2}}{p_{0}-p_{i}}
$$

This number becomes higher than 1 at $\omega=4000 \mathrm{rpm}$, corresponding to the appearance of centrifugal effects.

\subsection{Coupled results}

In this second set of simulations, the fluid at the inner radius (LP fluid) is different from the fluid at the outer radius (HP fluid). The solution procedure is thus a little bit more complex. Indeed, it necessary to solve the transport Eq. (7) at each step in order to update the local viscosity and density of the mixture. The computation time is thus increased compared to the first case where the two equations were not coupled.

The difference between the two fluids is the amount of gas content ( $\lambda$ in Eq. (2)). It has a direct impact on the local density and viscosity as indicated on Table 3 . The fluid at the inner radius obviously contains more air because this is the atmosphere side. The unknown in the transport equation (7) is thus $\lambda$ instead of $c$.

The results are presented in Figs. 8 to 12. Figure 8 presents the fluid pressure distribution at four different rotating speed values. For the three lowest velocity values, the pressure distributions are very similar to those obtained in the uncoupled case: a hydrodynamic pressure peak is generated as well as a sub-ambient pressure zone. However, for this highest speed value, the pressure field is completely different. The pressure peak becomes narrow and of weak amplitude and moves toward the outer radius.

The friction torque and the centerline clearance are presented as a function of the rotating speed on Fig. 9. The solids lines correspond to the coupled results. The previous simulations results are plotted with a dashed line. The values obtained with both simulations are superimposed to a rotating speed up to about $4000 \mathrm{rpm}$. Below this speed a typical Stribeck curve is obtained and the film thickness increases gradually with the angular velocity. Above $4000 \mathrm{rpm}$, a drastic decrease in film thickness is observed coupled with a significant raise of the friction. The film thickness drops of about $60 \%$.

An explanation of this behavior is found when examining Fig. 10. The distribution of the gas mass fraction at four different speed values is presented. For the three lowest speed values, the invasion of the LP fluid is limited to a zone close to the inner radius. At the highest speed, the LP fluid covers almost all the sealing interface. Since the characteristics of the LP fluid are

Table 3 Additional data for the coupled simulations

\begin{tabular}{|l|l|l|}
\hline Parameter & At the inner radius & At the outer radius \\
\hline Gas mass fraction $\lambda$ & $10^{-2}$ & $10^{-5}$ \\
\hline Pressure $p(\mathrm{~Pa})$ & $10^{5}$ & $2 \times 10^{5}$ \\
\hline Density $\rho\left(\mathrm{k} / \mathrm{m}^{3}\right)$ & 81.7 & 995 \\
\hline Viscosity $\mu(\mathrm{Pa} \cdot \mathrm{s})$ & $6.47 \times 10^{-4}$ & $9.99 \times 10^{-4}$ \\
\hline
\end{tabular}




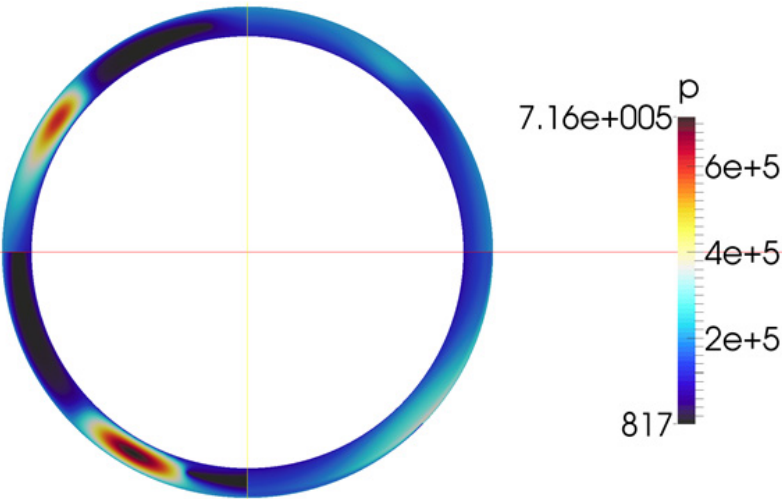

Fig 8 Pressure distribution $(\mathrm{Pa})$ in the fluid film domain when $\omega=$, top right $1 \mathrm{rpm}$, top left $32 \mathrm{rpm}$, bottom left $1024 \mathrm{rpm}$, bottom right $16384 \mathrm{rpm}$

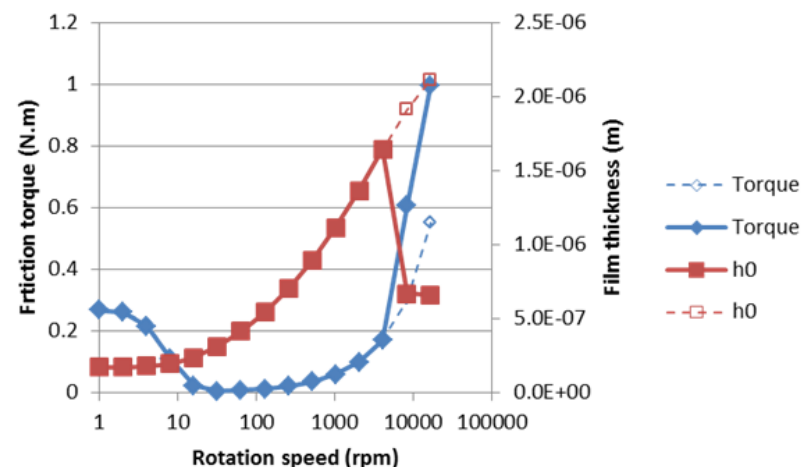

Fig 9 Friction torque and centerline clearance $h_{0}$ as a function of the rotation speed. The dashed lines correspond to results obtained with the uncoupled simulations

very different to those of the HP fluid, the seal behaves differently when it is lubricated with LP rather than HP fluid. This is the reason why a significant film thickness reduction is found at high speed.

The dimensionless area covered by the LP fluid as well as the leakage mass flow are plotted as a function of the rotation speed in Fig. 11. The dashed lines correspond to simulation results obtained with the previous uncoupled simulations. The coupled and uncoupled cases lead to similar results when the speed is lower than 4000 $\mathrm{rpm}$. When the velocity is higher, the centrifugal inertia becomes significant which help the LP fluid to enter the contact. Different behaviors are observed in this situation because the LP fluid has a different composition in the present simulations. However, a sharp decrease and an inversion of the mass flow is observed coupled with a fast increase of the area covered by the LP fluid. The latter can reach $91 \%$ at the highest speed value.

Finally, the fluid density distribution is presented in Fig. 12 for four different speed values. For 1, 32 and $1024 \mathrm{rpm}$, the LP fluid invasion is clearly visible close to the inner radius because of its low density compared to the HP fluid. When the angular velocity is set at 32 and $1024 \mathrm{rpm}$, a second low density zone corresponding to

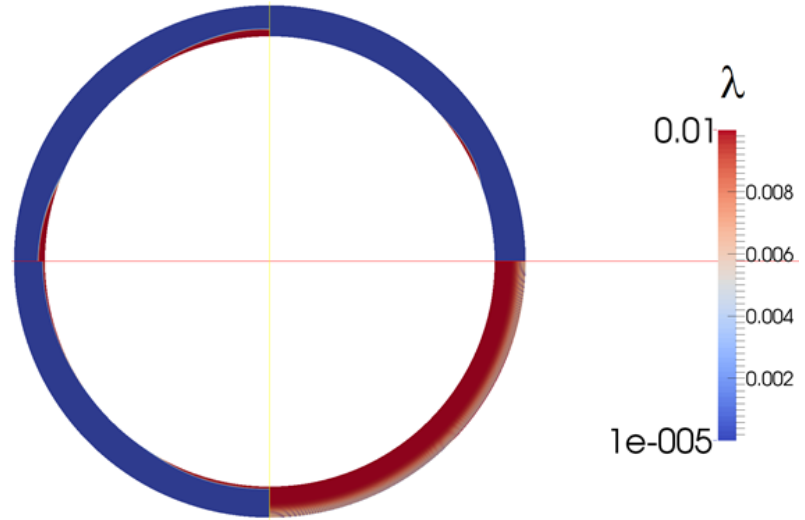

Fig 10 Gas mass fraction distribution in the fluid film domain when $\omega=$, top right $1 \mathrm{rpm}$, top left $32 \mathrm{rpm}$, bottom left $1024 \mathrm{rpm}$, bottom right $16384 \mathrm{rpm}$

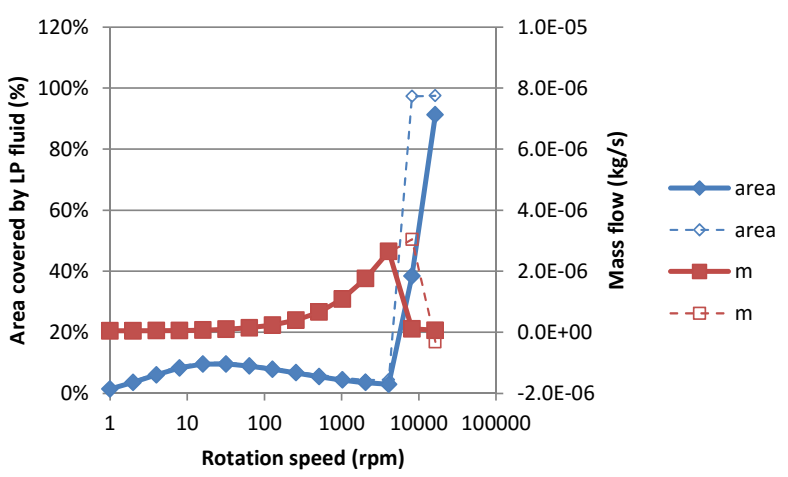

Fig 11 Dimensionless area covered by the LP fluid and the leakage mass flow as a function of the rotation speed. The dashed lines correspond to results obtained with the uncoupled simulations

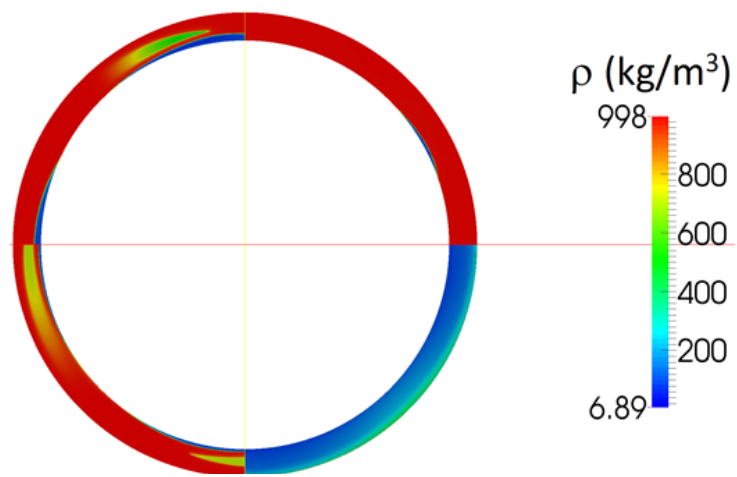

Fig 12 Density distribution $\left(\mathrm{kg} / \mathrm{m}^{3}\right)$ in the fluid film domain when $\omega=$, top right $1 \mathrm{rpm}$, top left $32 \mathrm{rpm}$, bottom left $1024 \mathrm{rpm}$, bottom right $16384 \mathrm{rpm}$

cavitation can be observed. For the highest speed, a very smooth density variation is obtained. Because of the gas like behavior of the LP fluid, no cavitation zone can be observed in this case. A density gradient is obtained in the radial direction because of a small amount of HP fluid entering the contact. 


\section{Conclusion}

This paper presented a method to numerically study reverse flows in mechanical face seals. The fluid is described by a homogenous mixture. The fluid flow is governed by the Reynolds equation and a transport equation is used to determine the fluid component distribution.

The model has been applied to a wavy seal where sub-ambient pressure zones are generated due to cavitation. Because of these low pressure zones, the fluid located at the low-pressure side is suck into the contact. It can invade an area of about $10 \%$ of the sealing interface. This area first increases with speed reaches a maximum and then decreases.

If the rotating speed is high enough to generate significant centrifugal inertia $\left(\operatorname{Re}_{C}{ }^{*}\right.$ higher than 1$)$, the direction of the mass flow can be inverted. The low pressure fluid occupies then almost all the sealing area. In this case, significant behavior variations can be observed. In the present work, the film thickness is decreased of about $60 \%$ whereas the friction increases.

\section{References}

[1] Johnson, R. and Schoenherr, K., "Wear Control Handbook Seal Wear," ASME, 1980, 727-753.

[2] Denny, D., "Some Measurements of Fluid Pressures Between Plane Parallel Thrust Surfaces with Special Reference to Radial-Face Seals," Wear, 4, 1961, 64-83.

[3] Nau, B., "Hydrodynamic Lubrication in Face Seals," Proc. of the $3^{\text {rd }}$ International Conference on Fluid Sealing, 1967, 73-120.

[4] Lebeck, A., "Principle and Design of Mechanical Face Seals," John Wiley \& Sons, Inc., New York, 1991.

[5] Muller, H. K., "Concepts of Sealing Mechanism of Rubber Lip Type Rotary Shaft Seals," Proc. of the $11^{\text {th }}$ international conference on fluid sealing. BHRA; 1987, 698-709.

[6] Wallis, G., "One-Dimensional Two-Phase Flow," Mc Graw-Hill, 1969.

[7] Szeri, A., "On the Flow of Emulsions in Tribological Contacts," Wear, 200, 1996, 353-364.

[8] Hughes, W., Winowich, N., Birchk, M. and Kennedy, W., "Phase Change in Liquid Face Seals," Journal of Lubrication Technology, 100, 1978, $74-80$.

[9] Yasuna, J. and Hughes, W., “A Continuous Boiling Model for Face Seals," Journal of Tribology, 112, 1990, 266-274.

[10] Migout, F., Brunetière, N. and Tournerie, B., "Study of the Fluid Film Vaporization in the Interface of a Mechanical Face Seal," Tribology International, 92, 2015, 84-95.
[11] Wang, T., Huang, W., Liu, Y., Liu, X. and Y, W., “A Homogeneous Phase Change Model for Two-Phase Mechanical Seals with Three-Dimensional Face Structures," Journal of Tribology, 136, 2014, 041708-11.

[12] Lebeck, A., "Experiments and Modeling of Zero Leakage Backward Pumping Mechanical Face Seals," Tribology Transactions, 51, 2008, 389-395.

[13] Brunetière, N. and Wang, Q., "A Simplified Mass-Conservating and Continuous Cavitation Model," Proceeding of the 2011 STLE annual meeting, 2011

[14] Socolofsky, S. A. and Jirka, G. H., "Mixing and Transport Processes in the Environment," Texas A \& M University, CVEN489-501, $5^{\text {th }}$ edition, 2005, https://ceprofs.civil.tamu.edu/ssocolofsky/cven489 /Book/Book.htm.

[15] Greenwood, J. A. and Williamson, J. B. P., "Contact of Nominally Flat Surfaces," Proceedings of the Royal Society (London), A295, 1966, 300-319.

[16] Bonneau, D., Huitric, J. and Tournerie, B., "Finite Element Analysis of Grooved Gas Thrust Bearings and Grooved Gas Face Seals," Journal of Tribology, 115, 1993, 348-354.

[17] Hernandez, P. and Boudet, J., "Modeling of the Behavior of Dynamical Gas Seals: Calculation With a Finite Element Method Implicitly Assuring the Continuity of Flow," IMechE, Part J, Journal of Engineering Tribology, 209, 1995, 195-201.

[18] Zirkelback, N. and San Andrès, L., "Effect of Frequency Excitation on Force Coefficients of Spiral Groove Gas Seals," Journal of Tribology, 121, 1999, 853-863.

[19] Zienkiewicz, O. and Taylor, R., "The Finite Element Method", Butterworth Heinemann, 2000, 3 - Fluid Dynamics.

[20] Brooks, A. and Hughes, T., "Streamline-Upwind / Petrov Galerkinn Methods for Advection Dominated Flows," Proc. of the $3^{\text {rd }}$ International Conference on FEM in Fluid Flows, 2, 1980, 283-292.

[21] Kelly, D., Nagasawa, S., Zienkiewicz, O. and Heinrich, J., "A Note on Upwinding and Anisotropic Balancing Dissipation in Finite Element Approximations to Convective Diffusion Problems," International Journal for Numerical Methods in Engineering, 15, 1980, 1705-1711.

[22] Minet, C., Brunetière, N., Tournerie, B. and Fribourg, D., "Analysis and Modelling of the Topography of Mechanical Seal Faces," Tribology Transactions, 53, 2010, 799-815.

[23] Brunetière, N. and Tournerie, B., "The Effect of Inertia on Radial Flows - Application to Hydrostatic Seals,” Journal of Tribology, 128, 2006, 566-574. 